\title{
Simulations of a micro-PET System based on Liquid Xenon
}

\author{
A. Miceli ${ }^{1}$, J. Glister ${ }^{1}$, A. Andreyev ${ }^{2}$, D. Bryman ${ }^{2}$, L.

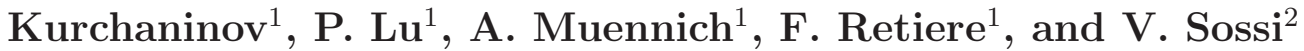 \\ 1 TRIUMF, 4004 Wesbrook Mall, Vancouver V6T 2A3, Canada \\ 2 Department of Physics and Astronomy, University of British Columbia, 6224 \\ Agricultural Road, Vancouver V6T 1Z1, Canada
}

\begin{abstract}
The imaging performance of a high-resolution preclinical microPET system employing liquid xenon as the gamma ray detection medium was simulated. The arrangement comprises a ring of detectors consisting of trapezoidal LXe time projection ionization chambers and two arrays of large area avalanche photodiodes for the measurement of ionization charge and scintillation light. A key feature of the LXePET system is the ability to identify individual photon interactions with high energy resolution and high spatial resolution in 3 dimensions and determine the correct interaction sequence using Compton reconstruction algorithms. The simulated LXePET imaging performance was evaluated by computing the noise equivalent count rate, the sensitivity and point spread function for a point source, and by examining the image quality using a micro-Derenzo phantom according to the NEMA-NU4 standard. Results of these simulation studies included NECR peaking at $1326 \mathrm{kcps}$ at $188 \mathrm{MBq}$ (705 kcps at $184 \mathrm{MBq}$ ) for an energy window of $450-600 \mathrm{keV}$ and a coincidence window of $1 \mathrm{~ns}$ for mouse (rat) phantoms. The absolute sensitivity at the center of the field of view was $12.6 \%$. Radial, tangential, and axial resolutions of ${ }^{22} \mathrm{Na}$ point sources reconstructed with a list-mode maximum likelihood expectation maximization algorithm were $\leq 0.8 \mathrm{~mm}$ (FWHM) throughout the field of view. Hot-rod inserts of $<$ $0.8 \mathrm{~mm}$ diameter were resolvable in the transaxial image of a micro-Derenzo phantom. The simulations show that a liquid xenon system would provide new capabilities for significantly enhancing PET images.
\end{abstract}




\section{Introduction}

Positron Emission Tomography (PET) is a functional medical imaging technique of increasing importance. Its power resides in the ability to investigate biological processes that are altered by disease and to trace radio-labeled molecules in organs. PET imaging can be used for early cancer screening, studying the pathology of illness, and to guide the development of new drugs.

Recently, several efforts were made to improve the sensitivity and spatial resolution of preclinical PET scanners by developing scintillation crystal-based detectors capable of measuring depth of interaction [1/5].

We are developing a novel high-resolution preclinical PET system using ionization and scintillation light signals from gamma ray interactions in liquid xenon (LXe). The Time Projection Chamber (TPC) [6] configuration is employed where ionization electrons are collected without gain on electrodes after drifting $11 \mathrm{~cm}$ under an applied electric field of $1-3 \mathrm{kV} / \mathrm{cm}$. Ionization from photon interactions can be localized in 3-D to $<1 \mathrm{~mm}$ because electron diffusion is small in LXe. Low diffusion also allows separation of individual photon interactions. Charge collection efficiency is high as long as the level of impurities in the LXe is sufficiently low (ppb level) [7,8]. Photon interactions also produce copious scintillation light in LXe (68000 photons/MeV at zero electric field) with time constants of $2.2 \mathrm{~ns}$ and $27 \mathrm{~ns}$, which is detected in our set-up by a set of Large Area Avalanche Photo-diodes (LAAPD) [9]; scintillation light is used to measure the interaction time with high resolution and contributes to the energy measurement. Furthermore, using both charge and scintillation light, excellent energy resolution $(<4 \%$ FWHM at $662 \mathrm{keV}$ ) has been reported [10]. LXe can be used to cover large detection volumes with high uniformity over the entire field of view (FOV) improving the detection sensitivity. Our previous studies on the use of LXe as a detection medium in PET were reported in [11]. The relevant properties of LXe are listed in Table 1.

In this paper, we describe a simulation of a LXe $\mu$-PET scanner and the Compton reconstruction algorithm developed for sequencing multi-interaction events. In addition, we present the simulated imaging performance of the LXePET system including sensitivity, scatter fraction, spatial resolution, and image quality evaluated according to the NEMA standard NU4 [12].

\begin{tabular}{lc}
\hline Property & Value \\
\hline Atomic number & 54 \\
Density & $3.1 \mathrm{~g} / \mathrm{cm}_{3}$ \\
Boiling point & $\mathrm{T}=165 \mathrm{~K}$ at $1 \mathrm{~atm}$ \\
Melting point & $\mathrm{T}=161 \mathrm{~K}$ at $1 \mathrm{~atm}$ \\
Photofraction at $511 \mathrm{keV}$ & $22 \%$ \\
Attenuation length at $511 \mathrm{keV}$ & $37 \mathrm{~mm}$ \\
Decay time & $2.2 \mathrm{~ns}, 27 \mathrm{~ns}$ \\
\hline
\end{tabular}

Table 1: Properties of liquid xenon. 


\section{Simulation Framework}

\subsection{Simulation model}

Figure 1 shows the planned configuration of the LXePET scanner consisting of twelve trapezoidal sectors arranged in a ring geometry. The inner bore has $10 \mathrm{~cm}$ dia. and $10 \mathrm{~cm}$ axial length. The liquid xenon is contained in a stainless-steel vessel thermally insulated by a vacuum space. Each sector is a LXeTPC viewed by two arrays of LAAPDs. The anode and cathode areas are $10 \mathrm{~cm} \times 9.2 \mathrm{~cm}$ and $10 \mathrm{~cm} \times 3.2 \mathrm{~cm}$, respectively, and the drift length is $11.2 \mathrm{~cm}$. Each APD array consists of 7 APDs with $16 \mathrm{~mm}$ dia., 9 APDs with $10 \mathrm{~mm}$ dia., and 8 APDs with $5 \mathrm{~mm}$ dia. Smaller APDs are used in the inner region to enhance the reconstruction where most of the events occur. Figure 2 shows the APD layout in one of the sectors.

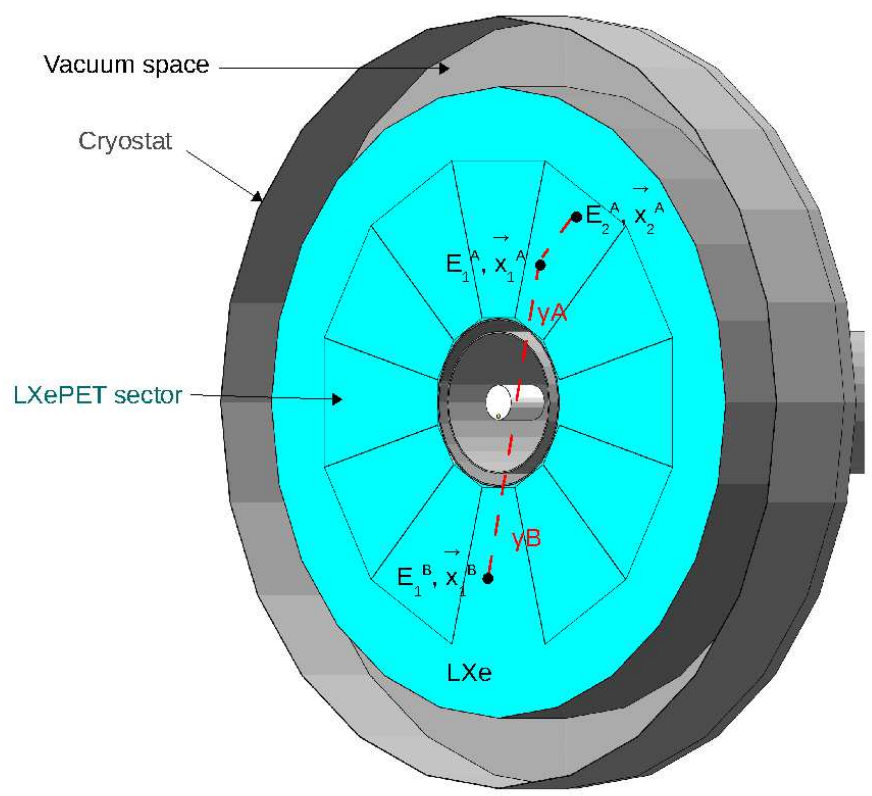

Figure 1: Simulated LXe PET system. The cryostat, the twelve LXePET sectors, the inner vessel filled with LXe (blue), and the mouse-like NEMA phantom are illustrated. The red dashed lines indicate a pair of annihilation photons which interact in the LXe. In this figure, photon A interacts twice in the LXePET sector, first via Compton scattering, then via the photoelectric effect. Photon B interacts only once via the photoelectric effect. Energy and 3-D position of each photon interaction are recorded by the TPC.

The simulation of the LXe prototype was carried out with the Geant4 simulation package [13. A positron emitter $\left({ }^{18} \mathrm{~F}\right.$ or ${ }^{22} \mathrm{Na}$ depending on the study) was simulated. Following the decay of the radioisotope, positrons with energy sampled from a continuous distribution of the beta decay process were generated and tracked until annihilation. To simulate the non-colinearity of the annihilation photons a new process was created and integrated in Geant4. The new process simulates the positron 


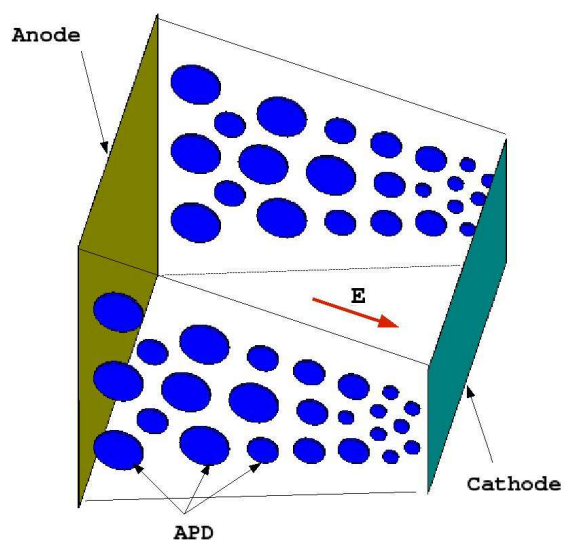

Figure 2: APD layout in one of the LXePET sectors.

annihilation in-flight according to the Geant4 annihilation process and replaces the Geant4 annihilation at rest with a model where the non-zero momentum of the electronpositron pair is taken into account. The interactions of the annihilation photons with the phantom and PET scanner were simulated with the low energy package of Geant4. Energy and 3D position of every photon interaction in the LXe detector were recorded. The numbers of ionization charges $N_{e-}^{i}$ and scintillation photons $S_{i}$ created in the interactions were calculated as [14]:

$$
\begin{aligned}
& N_{e-}^{i}=\frac{\left(1-F r^{*}\right) \times E_{i}^{G 4}}{15.6 e V} \\
& S^{i}=\frac{\left(v+F r^{*}\right) \times E_{i}^{G 4}}{15.6 e V}
\end{aligned}
$$

where $\mathrm{Fr}^{*}$ is the electron-ion recombination fraction, $E_{i}^{G 4}$ is the energy deposited in the interaction $i$ and $v=0.2$ [10] is the ratio of the number of excitons and ion pairs produced. The electron-ion recombination fraction $\mathrm{Fr}^{*}$ varies on an event-byevent basis. It was modeled as a Gaussian function centered at Fr $=0.24$ with width $\Delta F r=0.032$ [11]. Electronics and photo-detectors were not simulated directly. Instead, instrumental responses were parameterized in subsequent analyses as described in [14]. The parameters used in the simulation are listed in Table 2.

\subsection{Event Selection}

Coincidence events were selected using a two-step procedure. The first stage of the event selection simulated the response of the detector trigger using only the information from the scintillation light. Events producing less than 5000 scintillation photons (corresponding to approximately $180 \mathrm{keV}$ ) were rejected. For each photon of each annihilation pair passing the first selection stage, we calculated the energy from the scintillation light corrected for the solid angle using the information of the position from the charge measurement [14] and we used the resulting value to calculate the 


\begin{tabular}{lcc}
\hline Quantity & Symbol & Value \\
\hline Recombination factor & $\mathrm{Fr}$ & 0.24 \\
Fluctuation of the e-ion recombination & $\Delta F r$ & 0.032 \\
Position resolution & $\sigma_{\text {pos }}$ & $0.3 \mathrm{~mm}$ \\
Minimum two-hits separation distance & $\mathrm{d}$ & $1 \mathrm{~mm}$ \\
Electronic noise charge (APD 16mm) & $E N C_{A}^{16}$ & $5000 \mathrm{e}-$ \\
Electronic noise charge (APD 10mm) & $E N C_{A}^{10}$ & $2000 \mathrm{e}-$ \\
Electronic noise charge (APD 5mm) & $E N C_{A}^{5}$ & $500 \mathrm{e}-$ \\
APD quantum efficiency & $\mathrm{QE}$ & $80 \%$ \\
APD gain & $\mathrm{G}$ & 500 \\
APD excess noise factor & $\mathrm{F}$ & 2.5 \\
TPC electronic noise & $E N C_{Q}$ & $600 \mathrm{e}-$ \\
Charge detection threshold & $T_{C}$ & $1800 \mathrm{e}-$ \\
\hline
\end{tabular}

Table 2: Simulation parameters.

light-charge combined energy as described in 10. Events with combined energy 450$600 \mathrm{keV}$ were kept. The first interaction points defining the lines of response (LOR) of the selected events were stored in a list-mode format. The Compton reconstruction algorithm described in section 2.3 was used to find the first interaction point for multisites events.

\subsection{Compton Reconstruction Algorithm}

When a photon interacts in the detector, it can Compton scatter multiple times before being photo-absorbed. A $511 \mathrm{keV}$ photon is roughly three times more likely to Compton scatter than be photo-absorbed when it first interacts in LXe. The simplest interaction configuration is the 1-1 case in which the detector registers only 1 discernible interaction point for each of the two photons, corresponding to photo-absorption without scattering. Practically, however, multi-hit scenarios such as 1-2, 1-3, 2-2, etc. are more common, and must be taken into account, as they contribute to blurring of the image due to ambiguity in the location of the first interaction point. The goal of the Compton reconstruction algorithm is to sort through all the possible scattering sequences, determine the path that is the most probable, and define the most likely first interaction point and its associated line of response.

For each pair of photons interacting $M-N$ times in the detector, with $M$ representing photon 1 and $N$ photon 2 , and $M \leq N$, there are $M ! N$ ! number of possible interaction sequences. For each sequence, a LOR check is first performed, determining whether the trajectory passes through the phantom. Then, if the sequence was found to be viable, Compton kinematics were used to compute a test statistic score associated with the sequence.

The Klein-Nishina formula determines the scattering angle based on the energy deposited:

$$
\cos \left(\theta_{E}\right)=1+m c^{2} \times\left(E_{\gamma i}^{-1}-E_{\gamma i+1}^{-1}\right)
$$


where $E_{\gamma i}$ is the photon energy before the $i^{\text {th }}$ step given by:

$$
E_{\gamma i}=E_{\gamma 1}-\sum_{j=1}^{i-1} d E_{j}
$$

$\mathrm{m}$ is the electron mass, $\theta_{E}$ is the Compton scattering angle, $d E_{j}$ is the energy deposited at the $j^{\text {th }}$ step, and $E_{\gamma 1}=511 \mathrm{keV}$ is the energy of the photon before it reaches the detector. Alternatively, the scattering angle $\theta_{G}$ based on the position of interaction site is calculated as:

$$
\cos \left(\theta_{G}\right)=\frac{\overrightarrow{u_{i}} \cdot \overrightarrow{u_{i+1}}}{\left|\overrightarrow{u_{i}}\right|\left|\overrightarrow{u_{i+1}}\right|}
$$

where $\overrightarrow{u_{i}}=\left(x_{i}-x_{i-1}, y_{i}-y_{i-1}, z_{i}-z_{i-1}\right)$.

For each candidate interaction site, we could, in principle, determine if the sequence was the correct one by comparing the scattering angles computed using the energy deposited $\left(\theta_{E}\right)$ with the observed scattering angles given the geometric distribution of interaction sites $\left(\theta_{G}\right)$. In the ideal situation, the difference would be zero.

The ability to resolve the correct sequence, however, depends on the position and energy resolution of the system. A statistical weighting was used to account for instrumental resolution limits:

$$
\chi^{2}=\sum_{i=1}^{N-1} \frac{\left(\cos \left(\theta_{E}\right)_{i}-\cos \left(\theta_{G}\right)_{i}\right)^{2}}{\Delta \cos \left(\theta_{E}\right)_{i}^{2}+\Delta \cos \left(\theta_{G}\right)_{i}^{2}}
$$

where the error terms are defined as [15]:

$$
\Delta \cos \left(\theta_{E}\right)_{i}^{2}=m^{2} c^{4} \times\left(\frac{\sigma_{d E_{i}}^{2}}{E_{\gamma i}^{4}}+\sigma_{E \gamma i+1}^{2} \times\left(E_{\gamma i}^{-2}-E_{\gamma i+1}^{-2}\right)^{2}\right)
$$

and

$$
\Delta \cos \left(\theta_{G}\right)_{i}^{2}=\sigma_{\text {pos }}^{2} \times\left(\sigma g_{i, x}+\sigma g_{i, y}+\sigma g_{i, z}\right)
$$

where

$$
\sigma \vec{g}_{i}=\left(\frac{\overrightarrow{u_{i+1}}}{\left|\overrightarrow{u_{i}}\right| \cdot\left|\overrightarrow{u_{i+1}}\right|}-\frac{\overrightarrow{u_{i}} \times \cos \left(\theta_{G}\right)}{\left|\overrightarrow{u_{i}}\right|^{2}}\right)^{2}+\left(\frac{\overrightarrow{u_{i}}}{\left|\overrightarrow{u_{i}}\right| \cdot\left|u_{i+1}\right|}-\frac{\overrightarrow{u_{i+1}} \times \cos \left(\theta_{G}\right)}{\left|u_{i+1}\right|^{2}}\right)^{2}
$$

The error on the energy deposited at the $i^{t h}$ step, $\sigma_{d E_{i}}$, and the error on the photon energy after the interaction step $i, \sigma_{E \gamma i+1}$, are given by:

$$
\begin{aligned}
& \sigma_{d E_{i}}^{2}=E N C_{Q}^{2}+\Delta F r^{2} \times d E_{i}^{2} \\
& \sigma_{E \gamma i+1}^{2}=i \times E N C_{Q}^{2}+\Delta F r^{2} \times \sum_{j=1}^{i} d E_{j}^{2}
\end{aligned}
$$

Finally, the viable sequence with the lowest test statistic score was chosen by the reconstruction algorithm, and the associated LOR defined and recorded. If no suitable interaction sequence was found, the event was discarded. This reconstruction technique is similar to the one used in [15, 16] modified for PET applications. 


\subsection{Pile-up}

At high rates, fast scintillation light signals are used to roughly $\left(1 \mathrm{~cm}^{3}\right)$ localize the event in order to match correctly the light signal with the slowly drifting charge. Pileup of events can occur in the small volume determined by the light localization region and may contribute to the count losses. In order to improve the count rate capability of the LXePET system, a pile-up event recovery method based on energy balance and proximity to the light signal was developed. The efficiency of the algorithm was found to be $99 \%, 95 \%$, and $89 \%$ for 2, 3, and 4 -events of simulated pile-up. The fraction of pile-up events was evaluated by simulating a mouse and a rat phantom filled with water and ${ }^{18} F$. The time of each decay was simulated using a Poisson distribution. The count rate correction factor for the pileup with the recovery method, $\epsilon_{p}$, is given by:

$$
\epsilon_{p}=f_{s}+\sum_{k=2}^{4} f_{e}^{k} \times \mu^{k}
$$

where $f_{s}$ is the fraction of pile-up free events, $f_{e}^{k}$ is the fraction of k-events pileup, and $\mu^{k}$ is the efficiency of the pile-up recovery method for k-events pileup.

\subsection{Detection Rate Calculation}

The output of the simulation consisted of interaction steps for two types of events: singles where only one of the two photons interacted with the detector and double events where both photons reached the detector. These data are source activity independent and do not contain random coincidence events. In order to simulate the count rate performance of the LXe detector, the detection rates at different source activities and instrumental parameters, such as dead time and coincidence window, had to be calculated. Also, count losses due to the pile-up of events in the TPC were taken into account.

The calculation of detection rates was done by Poisson statistical modeling, taking into account the probability of each interaction type, and assuming that only events with exactly two emitted photons detected were selected. Given the trigger probabilities of detecting zero $\left(P_{0}\right)$, one $\left(P_{1}\right)$, and two $\left(P_{2}\right)$ photons from annihilation, the trigger rates for true and scatter events $C_{2,0}$ and for random events $C_{2 r, 0}$ for a given source activity, $\mathrm{A}$, and coincidence window, $\Delta t$, can be computed:

$$
\begin{aligned}
& C_{2,0}(A)=\frac{1}{\Delta t} \sum_{k=1}^{\infty} \frac{e^{-\lambda} \lambda^{k}}{k !} P_{2} P_{0}^{k-1}, \text { where } \lambda=A * \Delta t \text { and } \\
& C_{2 r, 0}(A)=\frac{1}{\Delta t} \sum_{k=2}^{\infty} \frac{e^{-\lambda} \lambda^{k}}{k !} P_{1}^{2} P_{0}^{k-2}
\end{aligned}
$$

Coincidence windows of 1,3 , and 6 ns were considered in these studies. The count rate for true and scatter events $C_{2}(A)$ and for randoms $C_{2 r}(A)$ are calculated as:

$$
C_{2}(A)=\frac{C_{2,0}}{1+C_{\text {total }, 0} \tau} \epsilon_{2} \times \epsilon_{p}^{2},
$$




\begin{tabular}{llcc}
\hline & & $\begin{array}{c}\text { Mouse-Like } \\
\text { Phantom }\end{array}$ & $\begin{array}{c}\text { Rat-Like } \\
\text { Phantom }\end{array}$ \\
\hline Scenario & {$\left[P_{0}\right](\%)$} & 41.5 & 40.8 \\
& {$\left[P_{1}\right](\%)$} & 36.3 & 46.6 \\
& {$\left[P_{2}\right](\%)$} & 22.2 & 12.6 \\
Efficiency & $\epsilon_{2}(\%)$ & 43.3 & 33.5 \\
& $\epsilon_{2 r}(\%)$ & 3.39 & 6.26 \\
\hline
\end{tabular}

Table 3: Trigger probabilities for zero, one, two photons $\left(P_{0}, P_{1}, P_{2}\right)$, and probabilities $\left(\epsilon_{2}, \epsilon_{2 r}\right)$ of a triggered event to pass the event selection for non-random and random events.

$$
C_{2 r}(A)=\frac{C_{2 r, 0}}{1+C_{\text {total }, 0} \tau} \epsilon_{2 r} \times \epsilon_{p}^{2}
$$

where $\tau$ is the instrumental dead time, $\epsilon_{p}$ is the count rate correction factor for the pile-up (Eq 12), and $\epsilon_{2}$ and $\epsilon_{2 r}$ are the probabilities of a triggered event to pass the event selection criteria. $C_{\text {total }, 0}$ is the total trigger rate including random coincidences. The ratios $\epsilon_{2}$ and $\epsilon_{2 r}$ depend on the combined energy resolution and energy window threshold, as well as on the event reconstruction strategy used. They are calculated for each data set (simulated true plus scatter data set and random data set) as the number of events which have combined energy within the 450-600 keV energy window and define a LOR which passes through the phantom, divided by the number of triggered events. The random set was generated by combining single unrelated events in pairs. The first stage trigger probabilities for zero, one, two photons detection, and 2nd-stage event selection efficiencies are given in Table 3. The trigger probability of detecting one or two photons is $60 \%$ for both the mouse and rat phantoms. The probability of detecting two photons depositing more than $180 \mathrm{keV}$ is $22 \%$, significantly higher for the mouse phantom than $13 \%$ found for the rat phantom due to the smaller amount of scattering produced by the mouse phantom. The amount of scattering is related to the size of the phantom.

Once the two final detection rates were calculated, a rate dependence could be applied to the output of the Geant 4 simulation. This was done by scaling the simulated double (true and scatter) and random events (pair-wise combinations of single events) to obtain the total detection rate $C_{2}(A)+C_{2 r}(A)$. This scaling approach allowed us to use a single large set of simulation data to compute the behavior of the detector and its performance at various resolution limits and activities without the need to re-simulate under different detector parameters.

\subsection{Image reconstruction}

In order to preserve the high resolution spatial information contained in the data produced by the LXePET scanner, we reconstructed the point source data and the micro-Derenzo phantom with a list-mode reconstruction algorithm. The main 
advantages of list-mode data reconstruction over rebinned data reconstruction are preservation of the maximum sampling frequency, and faster reconstruction for lowstatistics frames. Data reconstructed with histogram-mode methods are compressed in the axial and radial directions to reduce the sinogram size and to accelerate the reconstruction with a consequent loss of axial and transaxial resolution [17. This effect is particularly evident moving away from the axial axis in the transaxial plane. List-mode methods reconstruct the data event-by-event without the need of binning the data into space and time intervals thereby preventing information losses. The information preserving characteristic of list-mode reconstruction methods is particularly useful for high spatial and temporal resolution PET systems [18]. The computational time of histogram-mode reconstruction methods depends on the number of line of responses in the sinogram, whereas reconstruction time of list-mode methods depends only on number of events recorded. List-mode methods are therefore preferred for high resolution scanners where the number of line of responses can be much higher than the number of recorded events [17]. List-mode image reconstruction methods are also favorable in time-of-flight PET [19], motion corrected PET [20, and dynamic and gated PET [21]. We used a 3D list-mode image reconstruction algorithm for PET based on the maximum likelihood expectation maximization (MLEM) approach [22]. As in [23] and [24] each detected LOR was considered as a unique projection bin with the number of counts in each projection bin $g_{i}$ equal to 1 . Using notations $f_{j}^{n}$ and $f_{j}^{n+1}$ for the intensity vectors in voxel $j$ for step $n$ and the next $n+1$ iteration estimates, the iteration step for the list-mode MLEM algorithm is equal to:

$$
f_{j}^{n+1}=\frac{f_{j}^{n}}{s_{j}} \sum_{i} p_{j i} \frac{1}{\sum_{k} p_{i k} f_{k}^{n}}
$$

where $p_{i j}$ is the value of the system matrix describing the probability that a given emission event $i$ originates from a certain voxel $j, s_{j}$ is the sensitivity value for voxel $j$. The list-mode MLEM used on-the-fly ray-driven forward and-back projection with bilinear interpolation [25]. We used 20 MLEM iterations for the ${ }^{22} \mathrm{Na}$ point sources and 100 MLEM iterations for the Derenzo phantom. The voxel size was $0.15 \times 0.15 \mathrm{x}$ $0.15 \mathrm{~mm}^{3}$ and the image size was $360 \times 360 \times 360$ voxels. The reconstruction time for point sources (5.5 million LORs on average) was less than 3 hours on an Intel Xeon 2.00 $\mathrm{GHz} \mathrm{CPU}$ (single core). The reconstruction speed of the list-mode MLEM algorithm can be further improved by using the ordered subsets (OS) approach [26] and parallel processing.

\subsection{Simulated Data}

The system performance was evaluated based on the National Electrical Manufacturers Association (NEMA) standards [12]. The only deviation from the NEMA protocol was the use of a list-mode MLEM reconstruction method instead of FBP reconstruction algorithm for the spatial resolution studies. As explained in section 2.6. we used the 
list-mode MLEM method in order to preserve the high resolution spatial information of the scanner.

Sensitivity. The sensitivity of the system was determined with a $\mathrm{Na}^{22}$ point source embedded in a $1 \mathrm{~cm}^{3}$ acrylic cube. The source was stepped axially over the axial length of the scanner. The data were rebinned using the SSRB.

Scatter fraction and count rate performance. The scatter fraction and count rate performance were obtained with a mouse and a rat-like phantoms. The mouse-like phantom was a $25 \mathrm{~mm}$ dia. and $70 \mathrm{~mm}$ length polyethylene cylinder with a $3.2 \mathrm{~mm}$ dia. hole drilled at a radial distance of $10 \mathrm{~mm}$. A simulated $3.2 \mathrm{~mm}$ dia. $60 \mathrm{~mm}$ long rod was filled with water and ${ }^{18} \mathrm{~F}$. The rat-like phantom was a $50 \mathrm{~mm}$ dia. and $150 \mathrm{~mm}$ length polyethylene cylinder with a $3.2 \mathrm{~mm}$ dia. hole drilled at a radial distance of 17.5

$\mathrm{mm}$. A simulated $3.2 \mathrm{~mm}$ dia. $140 \mathrm{~mm}$ long rod was filled with water and the ${ }^{18} \mathrm{~F}$. The data were rebinned using the SSRB.

Spatial resolution. The spatial resolution was obtained with the $N a^{22}$ point source used for the sensitivity studies. The source was placed at two axial positions 0 and $12.5 \mathrm{~mm}$ and five radial positions $0,5,10,15$, and $25 \mathrm{~mm}$. The simulated data were reconstructed with the list-mode MLEM iterative method.

Image quality: The image quality was studied with a micro-Derenzo phantom made from acrylic measuring $40 \mathrm{~mm}$ in dia. and $35 \mathrm{~mm}$ in length. Arrayed throughout the phantom were cylindrical rods of length $30 \mathrm{~mm}$ and diameters 1.6, 1.4, 1.2, 1.0, 0.8 and $0.6 \mathrm{~mm}$. The rods were offset radially by $7 \mathrm{~mm}$ from the phantom center and filled with water and ${ }^{18} \mathrm{~F}$. The rod-to-rod separation was set to twice the rod diameter. The simulated data were reconstructed with the list-mode MLEM iterative method.

\section{Analysis}

\subsection{Sensitivity}

The absolute sensitivity was calculated following the NEMA standard. A simulated ${ }^{22} \mathrm{Na}$ point source was used for this study. The point source was stepped axially through the scanner at $0.5 \mathrm{~mm}$ steps over an axial length of $100 \mathrm{~mm}$. One million ${ }^{22} \mathrm{Na}$ decays were simulated at each step. The total absolute sensitivity for mouse applications was calculated by summing the sensitivity for the sinograms which encompass the central 7 $\mathrm{cm}$. Since the axial extent of the scanner was less than the length of the rat phantom, we calculated the total absolute sensitivity for rat applications summing all the slices, as described in the NEMA standard. The absolute sensitivity at the center of the field of view (CFOV) for an energy window of $[450,600] \mathrm{keV}$ was $12.6 \%$. The sensitivity profile for all axial steps can be seen in Figure 3. The total absolute sensitivity for mouse and rat applications were $9.4 \%$ and $7.2 \%$. The total system sensitivity was $7.2 \%$. For comparison, typical values of the absolute sensitivity at CFOV range from $3.4 \%$ for the microPET FOCUS-220 with $7.6 \mathrm{~cm}$ axial FOV, 250-750 keV energy window, and $10 \mathrm{~ns}$ time window [27] to $9.3 \%$ for the Inveon system with $12.7 \mathrm{~cm}$ axial FOV, 250-625 keV 
energy window, and 3.4 ns time window [28] .

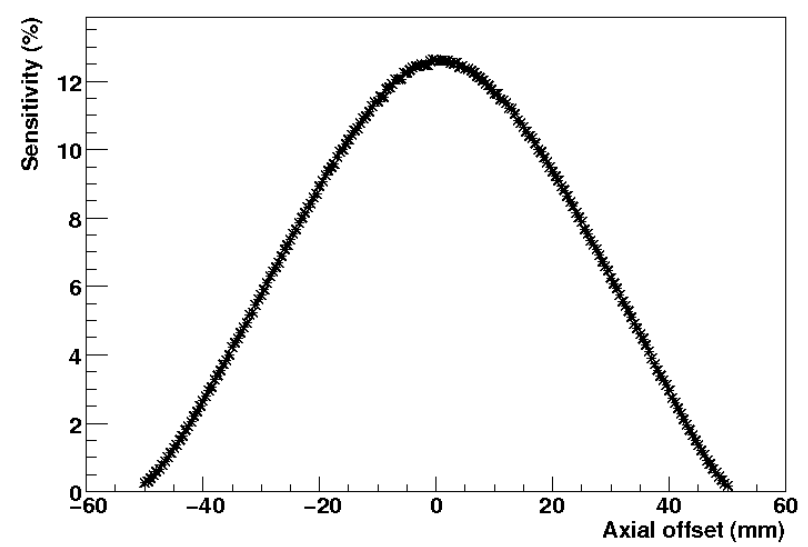

Figure 3: NEMA standard sensitivity profile for a ${ }^{22} \mathrm{Na}$ point source embedded in a 1 $\mathrm{cm}^{3}$ acrylic cube, measured at $0.5 \mathrm{~mm}$ axial steps. Energy window: $[450,600] \mathrm{keV}$.

\subsection{Scatter Fraction and Count Rate Performance}

The scatter fraction and noise equivalent count rate (NECR) studies were carried out using the rat-like and mouse-like phantoms following the NEMA protocol. For each phantom 50 million ${ }^{18} \mathrm{~F}$ decays were simulated. The list-mode simulated true plus scatter data set was arranged in sinograms (radial bin size $0.3 \mathrm{~mm}$ ) and oblique slices were combined into 2D projections using the SSRB method with a $1 \mathrm{~cm}$ slice thickness. For each sinogram, all pixels located farther than $8 \mathrm{~mm}$ from the edges of the phantom were set to zero. The profile of each projection angle was shifted so that the maximum value was aligned with the central pixel of the sinogram. All the angular projections were then summed to generate a sum projection. All counts outside the central $14 \mathrm{~mm}$ band were assumed to be scatter counts. To evaluate the scatter inside the $14 \mathrm{~mm}$ central band we used a linear interpolation. For each slice $i$, the number of scatter counts $C_{\text {scatt }, i}$ was given by the total scatter counts in the sinogram (outside and inside the central $14 \mathrm{~mm}$ band) divided by the number of pairs in the data set. The total event count $C_{T O T, i}$ is the sum of the pixels in the projections divided by the number of pairs in the data set. The scatter fraction is given by

$$
S F=\sum_{i=1}^{N \text { Slices }} C_{\text {scatt }, i} / C_{T O T, i}
$$

The mouse (rat) scatter fraction was $12.1 \%(20.8 \%)$, of which $4.9 \%$ (10.5\%) was due to scatter only and $7.2 \%(10.3 \%)$ was due to ambiguities in the Compton reconstruction algorithm. A future paper will deal with reducing the ambiguities. An example of Compton ambiguity involves multi-interaction events where one or both photons interact in only two locations and deposit the same amount of energy. To calculate the percentage 
of the scatter fraction due to Compton ambiguities we selected only true events in the simulation data set.

The random set was arranged in sinograms (radial bin size $0.3 \mathrm{~mm}$ ) and oblique slices were combined into $2 \mathrm{D}$ projections using the SSRB method with a $1 \mathrm{~cm}$ slice thickness. The number of random counts $C_{\text {random }, i}$ for each slice was the total counts in the random coincidence sinogram within $8 \mathrm{~mm}$ from the edges of the phantom divided by the number of pairs in the random set.

The noise equivalent rate for each slice was calculated as follows, where $C_{2}(A)$ and $C_{2 r}(A)$ are the rates previously calculated:

$$
N E C R_{i}(A)=\sum_{i=1}^{N \text { Slices }} \frac{\left(\left(C_{T O T, i}-C_{\text {scatt }, i}\right) \times C_{2}(A)\right)^{2}}{C_{T O T, i} \times C_{2}(A)+C_{\text {random }, i} \times C_{2 r}(A)}
$$

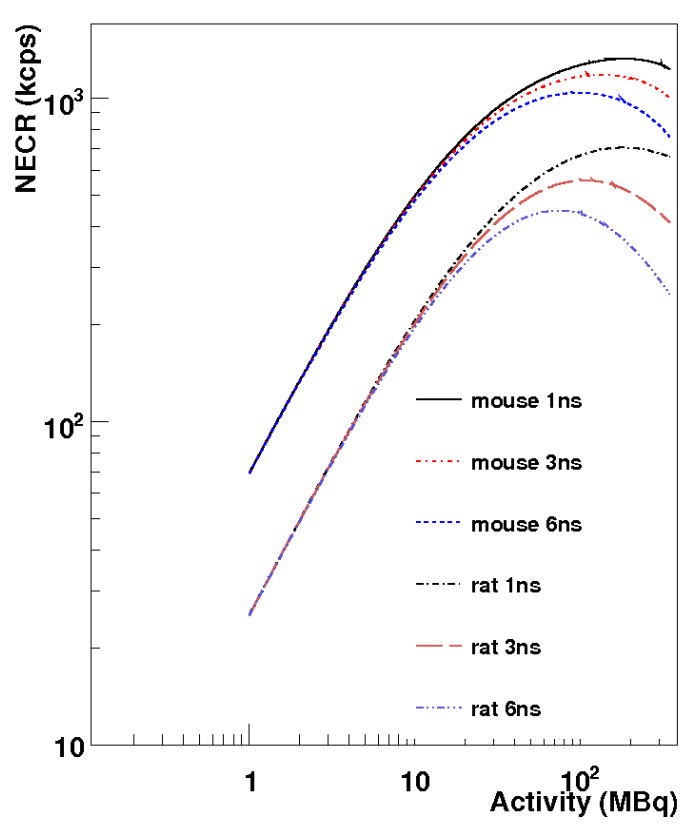

Figure 4: NECR vs. total activity, for mouse and rat phantoms and coincidence windows of 1,3 , and 6 ns. The dead time was $0.2 \mu$ s and the energy window was $[450,600] \mathrm{keV}$.

The NECR curves for mouse and rat phantoms are plotted in Figure 4 for 1, 3 and 6 ns coincidence windows. The scatter fractions $(S F)$, peak true counting rate $\left(R_{t, p e a k}\right)$, peak noise equivalent count rate $\left(R_{N E C, p e a k}\right)$, activity at which $R_{t, p e a k}$ is reached, and activity at which $R_{N E C \text {,peak }}$ is reached can be found in Table 4 for mouse and rat phantoms and the three coincidence windows with an energy window of $[450,600] \mathrm{keV}$. Figure 5 shows true, scatter, random, total counts, and NECR as a function of activity for the mouse-like phantom with coincidence window $6 \mathrm{~ns}$ and dead time $0.2 \mu \mathrm{s}$. The simulated results show a similar usable range of activity compared with commercial micro-PET systems (1670 kcps at $130 \mathrm{MBq}$ for a mouse phantom, a 350-625 keV energy window and 3.4 ns timing window - Inveon [28]). 


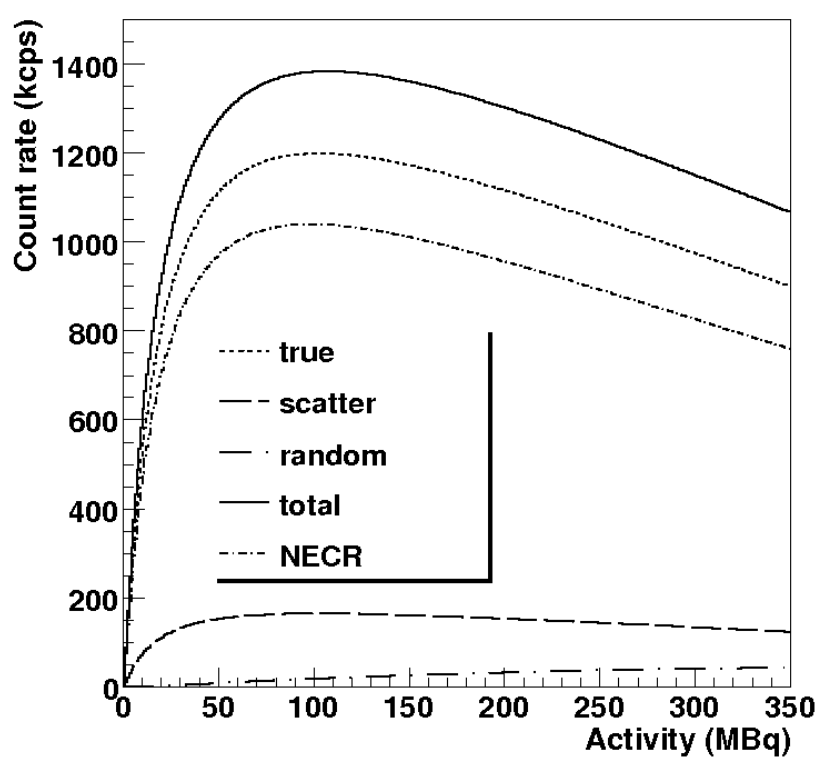

Figure 5: True, scatter, random, total counts, and NECR vs. total activity, for mouselike phantom and coincidence window 6 ns. The dead time was $0.2 \mu$ s and the energy window was $[450,600] \mathrm{keV}$.

\begin{tabular}{ccccccc}
\hline Phantom & $\begin{array}{c}\text { Coincidence } \\
\text { Window (ns) }\end{array}$ & SF (\%) & $\begin{array}{c}R_{t, \text { peak }} \\
(\mathrm{kcounts})\end{array}$ & $\begin{array}{c}R_{\text {NEC,peak }} \\
(\mathrm{kcounts})\end{array}$ & $\begin{array}{c}A_{t, \text { peak }} \\
(\mathrm{MBq})\end{array}$ & $\begin{array}{c}A_{\text {NEC,peak }} \\
(\mathrm{MBq})\end{array}$ \\
\hline Rat & 1 & 20.8 & 909 & 705 & 202 & 184 \\
& 3 & & 736 & 558 & 122 & 108 \\
\multirow{5}{*}{ Mouse } & 6 & & 605 & 450 & 86 & 75 \\
& 1 & 12.1 & 1515 & 1326 & 191 & 188 \\
& 3 & & 1359 & 1183 & 141 & 136 \\
& 6 & & 1200 & 1041 & 103 & 99 \\
\hline
\end{tabular}

Table 4: Scatter fraction and count rate performance for rat and mouse phantoms. The dead time was $0.2 \mu$ s and the energy window was $[450,600] \mathrm{keV}$.

\subsection{Spatial Resolution}

Spatial resolution was determined using the ${ }^{22} \mathrm{Na}$ point source with dia. $0.25 \mathrm{~mm}$ embedded in a $1 \mathrm{~cm}^{3}$ acrylic cube. A total of 50 million ${ }^{22} \mathrm{Na}$ decays were simulated and an energy window of $[450,600] \mathrm{keV}$ was used. It was assumed that the source activity would be low enough that random coincidences could be ignored. The source was placed at two axial positions: 0 and $12.5 \mathrm{~mm}$. Five radial positions were used for each axial position: 0, 5, 10, 15 and $25 \mathrm{~mm}$. The data were reconstructed with the list-mode MLEM iterative method (voxel size $0.15 \times 0.15 \times 0.15 \mathrm{~mm}, 20$ iterations). The point spread functions were formed by summing one-dimensional profiles parallel to the direction of measurement and within two FWHM of the orthogonal directions. The FWHM and FWTM values were calculated through linear interpolation between 


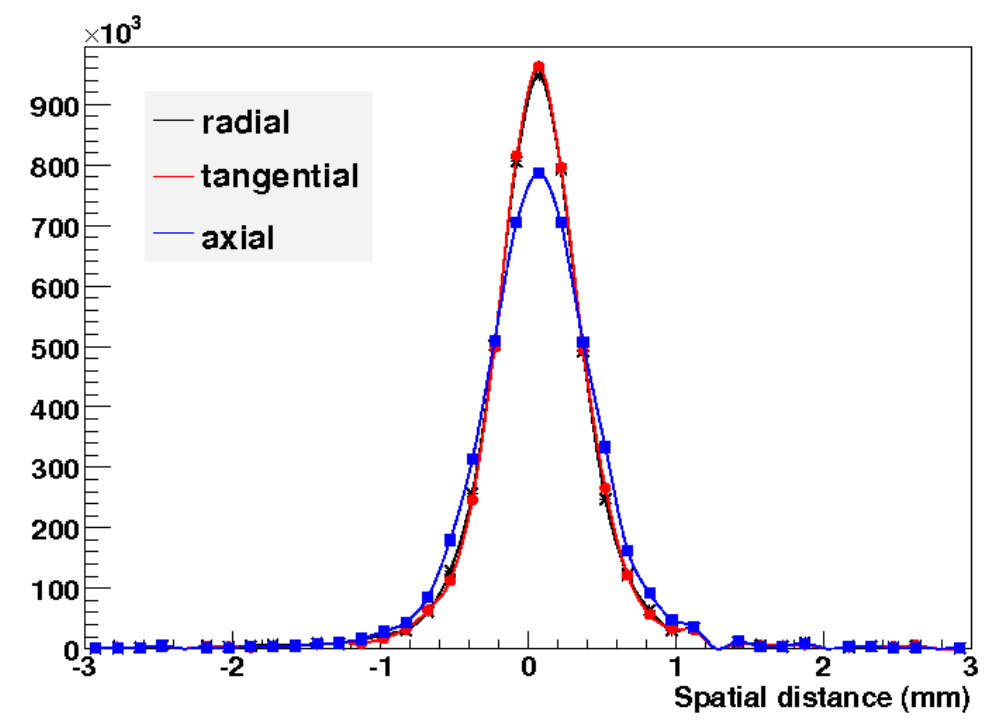

Figure 6: Point spread function in radial, tangential, and axial directions of a ${ }^{22} \mathrm{Na}$ point source at CFOV reconstructed with list-mode MLEM. Radial, tangential, and axial resolutions (FWHM) were $0.6 \mathrm{~mm}, 0.6 \mathrm{~mm}$, and $0.8 \mathrm{~mm}$.

adjacent pixels at one-half and one-tenth of the peak value in each direction. The point spread function for a point source at the CFOV is shown in Figure 6.

Radial, tangential and axial resolutions, reported as FWHM and FWTM, are given in Figures 7-8. At the CFOV radial, tangential, and axial FWHM resolutions of 0.6, 0.6, and $0.8 \mathrm{~mm}$ were found. At $25 \mathrm{~mm}$ radial and $12.5 \mathrm{~mm}$ axial offset, radial, tangential, and axial FWHM resolutions were $0.7,0.7$, and $0.8 \mathrm{~mm}$. The results show a uniform resolution $\leq 0.8 \mathrm{~mm}(\mathrm{FWHM})$ throughout the FOV in radial, tangential, and axial directions. At the CFOV, the 2DFBP gave the same results of the MLEM algorithm. For comparison, typical values of spatial resolution for conventional micro-PET systems are 1.3, 1.3, and $1.5 \mathrm{~mm}$ (microPET FOCUS-220 [27]). Also, the deterioration of the radial resolution towards the periphery of the FOV, which is common for crystals-based preclinical PET systems due to lack of DOI information, is absent in the LXePET.

\subsection{Image Quality Study}

Figure 9 shows a trans-axial slice (thickness $24 \mathrm{~mm}$ ) of the micro-Derenzo phantom with cylindrical rods of length $30 \mathrm{~mm}$ and diameters 1.6, 1.4, 1.2, 1.0, 0.8, and $0.6 \mathrm{~mm}$ reconstructed with the list-mode MLEM method (100 iterations). The voxel size was $0.15 \times 0.15 \times 0.15 \mathrm{~mm}$. No attenuation or scatter corrections were applied. The source activity was low enough that random coincidences could be ignored. Rods of diameter $0.6 \mathrm{~mm}$ to $1.6 \mathrm{~mm}$ are visible. 

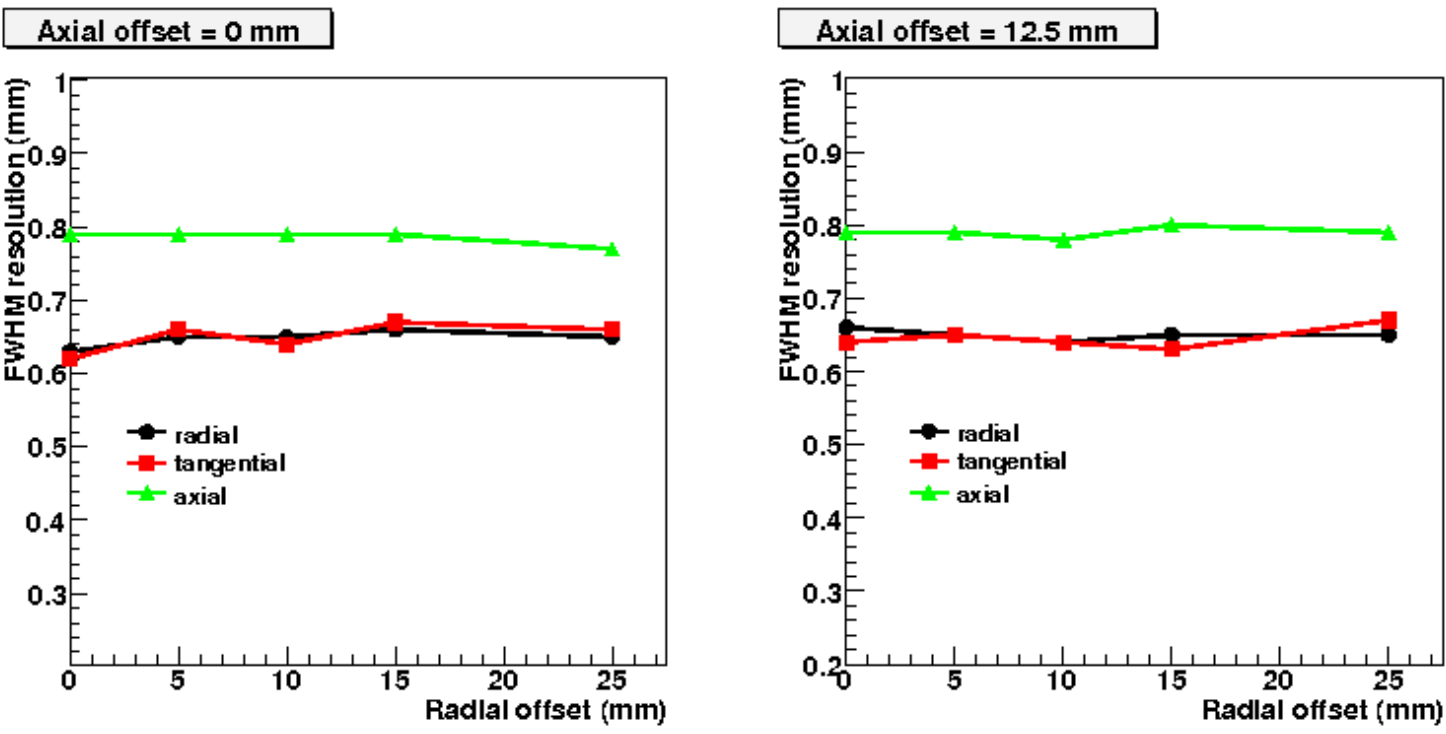

Figure 7: Radial, tangential, and axial resolution (FWHM) of a ${ }^{22} \mathrm{Na}$ point source reconstructed with list-mode MLEM.

Axial offset $=0 \mathrm{~mm}$

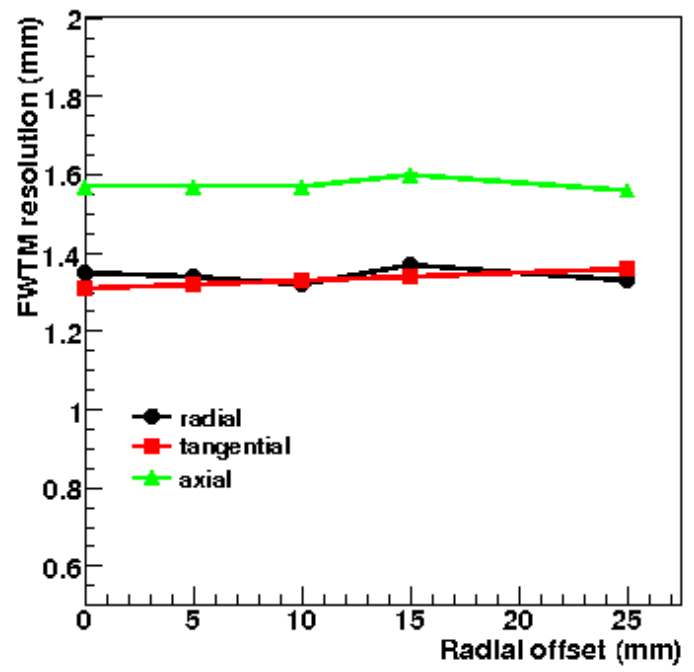

Axial offset $=12.5 \mathrm{~mm}$

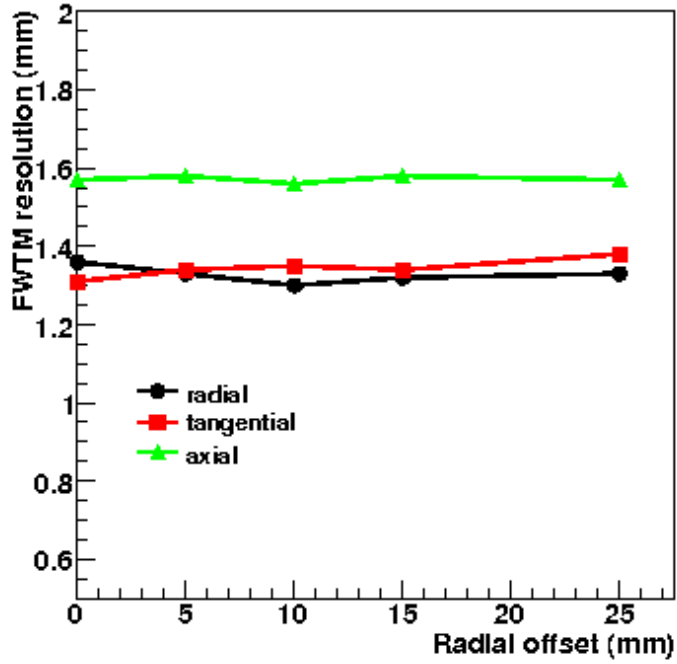

Figure 8: Radial, tangential, and axial FWTM of a ${ }^{22} \mathrm{Na}$ point source reconstructed with list-mode MLEM.

\section{Conclusion}

The potential imaging performance of a high resolution liquid xenon preclinical PET system was evaluated with Monte Carlo simulations. An event reconstruction algorithm was developed to handle multiple photon scatterings in liquid xenon, enabling us to refine the lines of response selections and reduce the event mispositioning introduced by scattered and random events which result in background noise. Using an energy 


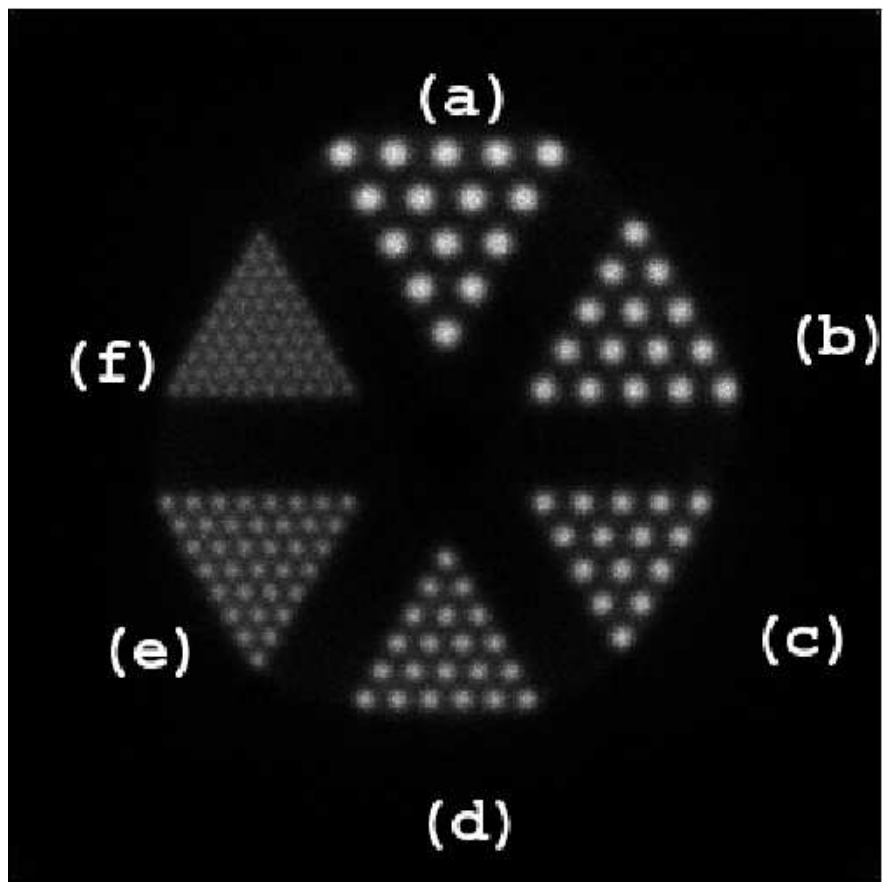

Figure 9: Micro-Derenzo phantom reconstructed using MLEM. Rod-to-rod separation is twice the rod diameter. Rod diameters: a $(1.6 \mathrm{~mm}), \mathrm{b}(1.4 \mathrm{~mm}), \mathrm{c}(1.2 \mathrm{~mm}), \mathrm{d}(1.0 \mathrm{~mm})$, $\mathrm{e}(0.8 \mathrm{~mm}), \mathrm{f}(0.6 \mathrm{~mm})$.

window $[450,600] \mathrm{keV}$ which is possible due to the high energy resolution, the results show that the LXePET system combines uniform high resolution radial, tangential, and axial position measurements throughout the field of view $(\leq 0.8 \mathrm{~mm}$ FWHM) with high sensitivity (12.6\% at CFOV) and the ability to reject scatter and random coincidences. The scatter fraction was found to be $20.8 \%$ (12.1\%), with associated peak NECR values of $1326 \mathrm{kcps}$ at $188 \mathrm{MBq}$ (705 kcps at $184 \mathrm{MBq}$ ) for mouse (rat)-like phantoms. These results show the potentially excellent imaging capabilities of the LXePET systems. Weighting schemes, where all available data are kept but each LOR is assigned a weight between 0 and 1, and filtering methods based on test statistic score computed with Compton kinematics will be investigated to further decrease noise in the images. Measurements are in progress to demonstrate the performance of the LXePET system described here.

\section{Acknowledgments}

This work was supported in part by NSERC, CIHR (CHRP Program), the Canada Foundation for Innovation, the University of British Columbia, and TRIUMF which receives founding via a contribution agreement with the National Research Council of Canada. 


\section{References}

[1] M. Bergeron, J. Cadorette, J.-F. Beaudoin, M.D. Lepage, G. Robert, V. Selivanov, M.-A. Tetrault, N. Viscogliosi, J.P. Norenberg, R. Fontaine, and R. Lecomte. Performance Evaluation of the LabPET APD-Based Digital PET Scanner. Nuclear Science, IEEE Transactions on, 56(1):10 $-16,2009$.

[2] Y. Tai, A. F. Chatziioannou, Y. Yang, R. W. Silverman, K. Meadors, S. Siegel, D. F. Newport, J. R. Stickel, and S. R. Cherry. MicroPET II: design, development and initial performance of an improved microPET scanner for small-animal imaging. Phys. Med. Biol., 48(11):1519-1537, 2003.

[3] P. Sempere Roldan, E. Chereul, O. Dietzel, L. Magnier, C. Pautrot, L. Rbah, D. Sappey-Marinier, A. Wagner, L. Zimmer, M. Janier, V. Tarazona, and G. Dietzel. Raytest ClearPET(TM), a new generation small animal PET scanner. Nucl. Instr. and Meth. A, 571(1-2):498- 501, 2007.

[4] J. Seidel, J.J. Vaquero, J. Pascau, M. Desco, C.A. Johnson, and M.V. Green. Features of the NIH atlas small animal PET scanner and its use with a coaxial small animal volume CT scanner. Proceedings, IEEE International Symposium on Biomedical Imaging, page 545 548, 2002.

[5] Yang Y, Wu Y, Qi J, St James S, Du H, Dokhale PA, Shah KS, Farrell R, and Cherry SR. A Prototype PET Scanner with DOI-Encoding Detectors. J Nucl Med, 49(7):1132 - 1140, 2008.

[6] J. N. Marx et al. The Time Projection Chamber. Phys. Today, 31N10:46, 1978.

[7] V. Chepel et al. Purification of Liquid Xenon and Impurity Monitoring for a PET Detector. Nucl. Instr. and Meth. A, 349:500-505, 1994.

[8] E. Conti et al. Correlated Fluctuations between Luminescence and Ionization in Liquid Xenon. Phys. Rev. B, 68:054201, 2003.

[9] M. Moszynski, M. Szawlowski, M. Kapusta, and M. Balcerzyk. Large area avalanche photodiodes in scintillation and X-rays detection. Nucl. Instr. and Meth. A, 485(3):504-521, 2002.

[10] E. Aprile et al. Observation of Anti-correlation between Scintillation and Ionization for $\mathrm{MeV}$ Gamma-Rays in Liquid Xenon. Phys. Rev. B, Issue 1, 76:014115.

[11] P. Amaudruz, D. Bryman, L. Kurchaninov, P. Lu, C. Marshall, J.P. Martin, A. Muennich, F. Retiere, and A. Sher. Simultaneous Reconstruction of Scintillation Light and Ionization Charge Produced by $511 \mathrm{keV}$ Photons in Liquid Xenon: Potential Application to PET. Nucl. Instr. and Meth. A, 607:668-767, 2009.

[12] NEMA Standards Publication NU4-2008: Performance Measurements of Small Animal Positron Emission Tomographs. National Electrical Manufacturers Association, Rosslyn, VA, 2008.

[13] S. Agostinelli et al. G4-a simulation toolkit. Nucl. Instr. and Meth. A, 506(3):250-303, 2003.

[14] A. Miceli, P. Amaudruz, F. Benard, D. Bryman, L. Kurchaninov, J. P. Martin, A. Muennich, F. Retiere, T. J. Ruth, V. Sossi, and A.J. Stoessl. Liquid Xenon Detectors for Positron Emission Tomography. Journal of Physics: Conference Series, in-press, arXiv:1104.1458v1.

[15] E. Aprile, A. Curioni, K.L. Giboni, M. Kobayashi, U.G. Oberlack, and S. Zhang. Compton imaging of $\mathrm{MeV}$ gamma-rays with the Liquid Xenon Gamma-Ray Imaging Telescope (LXeGRIT). Nucl. Instr. and Meth. A, 593(3):414-425, 2008.

[16] U.G. Oberlack et al. Compton Scattering Sequence Reconstruction Algorithm for the Liquid Xenon Gamma-Ray Imaging Telescope (LXeGRIT). arXiv:astro-ph/0012296.

[17] R. Arman. Statistical list-mode image reconstruction and motion compensation techniques in highresolution positron emission tomography. PhD thesis, The University of British Columbia, 2005.

[18] A. J. Reader. List-Mode EM Algorithms for Limited Precision High-Resolution PET Image Reconstruction. Int. J. Imaging Syst. Technol., 14:139-145, 2004.

[19] G. Pratx, S. Surti, and C. Levin. Fast List-Mode Reconstruction for Time-of-Flight PET Using Graphics Hardware. Nuclear Science, IEEE Transactions on, 58(1):105 -109, 2011.

[20] F. Lamare, M. J. Ledesma Carbayo, T. Cresson, G. Kontaxakis, A. Santos, C. Cheze Le Rest, A. J. Reader, and D. Visvikis. List-mode-based reconstruction for respiratory motion correction in PET using non-rigid body transformations. Physics in Medicine and Biology, 52(17):5187, 
2007.

[21] A. Rahmim, J.-C. Cheng, S. Blinder, M.-L. Camborde, and V. Sossi. Statistical dynamic image reconstruction in state-of-the-art high-resolution PET. Physics in Medicine and Biology, $50(20): 4887,2005$.

[22] L. A. Shepp and Y. Vardi. Maximum Likelihood Reconstruction in Positron Emission Tomography. IEEE Transactions on Medical Imaging, 1:113-122, 1982.

[23] H. H. Barrett, T. White, and L. C. Parra. List-Mode Likelihood. J. Opt. Soc. Am. A, 1997.

[24] L. Parra and H. H. Barrett. List-Mode Likelihood: EM Algorithm and Image Quality Estimation demonstrated on 2D PET. 17:228-235, 1998.

[25] A. Rahmim, T.J. Ruth, and V. Sossi. Study of a convergent subsetized list-mode EM reconstruction algorithm. Nuclear Science Symposium Conference Record, 2004 IEEE, 6:3978 $-3982,2004$.

[26] M. Hudson and R. Larkin. Accelerated Image Reconstruction using Ordered Subsets of Projection Data. IEEE Trans. Med. Imag, 13:601-609, 1994.

[27] Y-C Tai, A Ruangma, D Rowland, S Siegel, D. F. Newport, P. L. Chow, and R. Laforest. Performance Evaluation of the microPET Focus: A Third-Generation microPET Scanner Dedicated to Animal Imaging. J Nucl Med, 46:455-463, 2005.

[28] Q. Bao, D. Newport, M. Chen, D.B. Stout, and A. F. Chatziioannou. Performance Evaluation of the Inveon Dedicated PET Preclinical Tomograph Based on the NEMA NU-4 Standards. $J$ Nucl Med, 50:401-408, 2009. 